\section{Ashwell that ends well}

\section{By Lev Osherovich, Senior Writer}

The hemostatic system, or the balance between pro- and anticlotting factors, keeps blood poised on the edge of coagulation. Imbalances in the system caused by pathogens or inflammation can lead to uncontrolled bleeding or uncontrolled clotting and sepsis. A paper in Nature Medicine has identified a critical control knob in this mechanism: a hepatocyte surface protein called the Ashwell receptor. ${ }^{1}$

According to the authors, the findings offer a new mechanism underlying distributed intravascular coagulation (DIC), the systemic clotting triggered by bacterial sepsis.

\section{Blood sugar}

The mouse-based study was conducted by a Howard Hughes Medical Institute team, led by Jamey Marth, professor of cellular and molecular medicine at the University of California, San Diego.

The research uncovered a new function for the Ashwell receptor, which was identified over 30 years ago by NIH biochemist Gilbert Ashwell as the first C-type lectin, or calcium-dependent sugar-binding protein. ${ }^{2}$

The Ashwell receptor binds glycoproteins that lack sialic acid moieties on their sugar coating. Sialic acid is a signature sugar of animal glycoproteins that blocks bacteria and viruses from attaching to host cells. Glycoproteins that lack sialic acids are recognized as defective and are filtered out of the blood by the Ashwell receptor in the liver.

Marth's team found that the Ashwell receptor could also target platelets. In normal mice, platelets missing sialic acid rapidly disappeared from the blood, whereas these defective platelets remained in circulation longer in Ashwell receptor knockout mice, leading to excessive clotting.

In addition, Marth's team found that mice lacking the Ashwell receptor had higher circulating levels of von Willebrand factor (vWF), a clotpromoting glycoprotein, than wild-type controls.

The Ashwell receptor is thus a "clearance system" that filters out activated blood-clotting components, Marth told SciBX.

This clearance mechanism becomes critically important during bacterial infection, the team found. Ashwell receptor knockout mice had a markedly lower rate of survival after injection with Streptococcus pneumoniae than wild-type mice.

The findings suggest a new perspective on the hemostatic changes in sepsis, according to Marth. Previously, the drop in platelet levels in patients entering septic shock was thought to be caused directly by bacterial pathogens. Instead, he believes this could be the result of "a protective mechanism caused by the Ashwell receptor," which presumably removes defective platelets from circulation and thus reduces clotting.

Marth therefore believes stimulating Ashwell receptor activity could be a new therapeutic strategy to treat sepsis.

Indeed, Xigris drotrecogin alfa, a recombinant human protein $\mathrm{C}$ that blocks protease-activated receptor 1 (PAR1) and is marketed by Eli Lilly and Co., remains the only approved drug for sepsis.

One way to harness the Ashwell receptor might be to remove the sialic acid tags on circulating glycoproteins such as vWF and platelets, helping the receptors to sop them up before sepsis develops.

The way to do this, said Marth, is to introduce bacterial neuraminidase into the blood before sepsis sets in.

Many pathogens have evolved ways to evade the sialic acid barrier; in this case, neuraminidase enzymes lop off sialic acid moieties to reveal bacterial binding sites. S. pneumoniae, for example, uses neuraminidase to break down the glycoprotein barrier to cellular entry. ${ }^{3}$

However, by chopping off sialic acid, the bacterial enzyme actually helps the Ashwell receptor to recognize compromised proteins and thus, paradoxically, helps it clear these defective proteins from circulation.

Indeed, according to the study, S. pneumoniae strains lacking neuraminidase triggered more coagulation and death than did wild-type bacteria. Moreover, injecting recombinant neuraminidase into mice infected with neuraminidase-deficient S. pneumoniae reduced clotting and improved survival compared with what was seen in neuraminidase-free controls.

\section{Antisepsis}

John Hanover, chief of the Laboratory of Cell Biochemistry and Biology at the National Institute of Diabetes and Digestive and Kidney Diseases, told SciBX that the discovery of the Ashwell receptor's protective role in sepsis is "an unanticipated outcome."

Hanover, who collaborates with the 93-year-old Gilbert Ashwell, thinks the big surprise is that the Ashwell receptor mechanism is "extremely specific" for vWF and platelets. The receptor knockout mice are relatively healthy, suggesting that "the desialylation of serum glycoproteins is not a profound stress" to the organism, he said.

This is encouraging because sepsis therapeutics that target critical clotting factors such as thrombin or PAR1 can have undesired immunological side effects. ${ }^{4}$

Hanover suggested the next step would be to treat animals with isolated glycoprotein-binding fragments of the Ashwell receptor, akin to $\mathrm{mAbs}$, to "increase the clearance" of vWF and platelets.

He thinks this approach will be clinically relevant. "The receptors and glycoproteins are highly conserved among mammals," said Hanover. "It's reasonable to extrapolate to humans." 


\section{TARGETS \& MECHANISMS}

Robert Schaub, VP of preclinical discovery at Archemix Corp., noted the Ashwell receptor appears to decrease levels of vWF and platelets but does not block the activity of either. Depleting overall vWF levels could complement Archemix's strategy of blocking platelet activation because "these are entirely different mechanisms," he noted.

Archemix's ARC1779 aptamer blocks the interaction between vWF and platelets, a critical step in the cascade that leads to DIC. The compound is in Phase II trials to treat acute coronary syndrome (ACS) and thrombotic thrombocytopenic purpura (TTP), a hereditary defect in vWF degradation that leads to excessive clotting.

\section{Sepsis skeptics}

However, some industry researchers doubt that modulating coagulation through the Ashwell receptor could be an effective strategy to treat severe sepsis.

Yann Echelard, VP of research at GTC Biotherapeutics Inc., noted that Marth's findings may be relevant only in a subset of sepsis situations. "Less than $30 \%$ of cases of DIC are caused by S. pneumoniae," he said.

Echelard added that $S$. pneumoniae has additional virulence factors beyond neuraminidase, "so it is unclear that targeting the Ashwell receptor pathway would really influence the outcome of $S$. pneumoniae infections."

GTC's Atryn is in Phase III testing for DIC. The recombinant antithrombin is partnered with Leo Pharma A/S.

Arguing to the contrary, Marth told SciBX that he sees the neuraminidase-Ashwell receptor pathway as relevant to a variety of bacterial infections.

"Engaging the Ashwell receptor could have an even more significant impact" on sepsis caused by bacteria that do not rely on neuraminidase, such as Staphylococcus aureus, according to Marth. In staph infections, adding neuraminidase would make little difference to the bug, but might greatly increase the efficiency of clotting-factor clearance by the Ashwell receptor.

To test this hypothesis, Marth plans to treat mice with neuraminidase to remove sialic acid from clotting factors before infecting them with $S$. aureus.

The biggest question is whether stimulating the Ashwell receptor can stem systemic inflammation after infection and sepsis have already set in.

"It's important to be able to use this therapy after someone has entered sepsis," said Marth. He suspects that the treatment "might work a very significant time after the initiation of sepsis. It's really worth looking at this."

Marth also noted that other Ashwell receptor ligands could be important in preventing sepsis. "It remains to be shown whether platelets are in fact the most relevant targets," he said.

Marth said he has sought patents for the discoveries reported in the Nature Medicine paper and has had discussions with venture firms about launching a company based on this work.

\section{REFERENCES}

1. Grewal, P.K. et al. Nat. Med.; published online May 18, 2008;

doi:10.1038/nm1760

Contact: Jamey Marth, University of California, San Diego, La Jolla, Calif. e-mail: jmarth@ucsd.edu

2. Hudgin, R.L. et al. J. Biol. Chem. 249, 5536-5543 (1974)

3. Manco, S. et al. Infect. Immun. 74, 4014-4020 (2006)

4. Osherovich, L. SciBX 1(7), 1-3; March 13, 2008

\section{COMPANIES AND INSTITUTIONS MENTIONED}

Archemix Corp., Cambridge, Mass.

Eli Lilly and Co. (NYSE:LLY), Indianapolis, Ind.

GTC Biotherapeutics Inc. (NASDAQ:GTCB), Framingham, Mass.

Howard Hughes Medical Institute, Chevy Chase, Md.

Leo Pharma A/S, Ballerup, Denmark

National Institute of Diabetes and Digestive and Kidney Diseases,

Bethesda, Md.

University of California, San Diego, La Jolla, Calif. 